\title{
Some inequalities on the skew-spectral radii of oriented graphs
}

Guang-Hui Xu*

\section{"Correspondence:} ghxu@zafu.edu.cn

Department of Applied Mathematics, Zhejiang A \& F University, Hangzhou, 311300 , China

\section{第 Springer}

\begin{abstract}
Let $G$ be a simple graph and $G^{\sigma}$ be an oriented graph obtained from $G$ by assigning a direction to each edge of $G$. The adjacency matrix of $G$ is $A(G)$ and the skew-adjacency matrix of $G^{\sigma}$ is $S\left(G^{\sigma}\right)$. The adjacency spectral radius $\rho(G)$ of $G$ and the skew-spectral radius $\varrho\left(G^{\sigma}\right)$ of $G^{\sigma}$ are defined as the spectral radius of $A(G)$ and $S\left(G^{\sigma}\right)$ respectively.

In this paper, we firstly establish a relation between $\varrho\left(G^{\sigma}\right)$ and $\rho(G)$. Also, we give some results on the skew-spectral radii of $G^{\sigma}$ and its oriented subgraphs. As an application of these results, we obtain a sharp upper bound of the skew-spectral radius of an oriented unicyclic graph.

MSC: $05 \mathrm{C} 50 ; 15 \mathrm{~A} 18$
\end{abstract}

Keywords: oriented graph; skew-adjacency matrix; skew-spectral radius; unicyclic graph

\section{Introduction}

In this paper, $i$ will always denote an imaginary unit. The identity matrix is denoted by $I$ and the transpose of the matrix $A$ by $A^{T}$. Let $G$ be a simple graph with $n$ vertices. The adjacency matrix $A=A(G)$ is the symmetric matrix $\left[a_{j k}\right]_{n \times n}$, where $a_{j k}=a_{k j}=1$ if $v_{j} v_{k}$ is an edge of $G$, otherwise $a_{j k}=a_{k j}=0$. We call $\operatorname{det}(\lambda I-A)$ the characteristic polynomial of $G$, denoted by $P(G ; \lambda)$. The adjacency spectrum $S_{p}(G)$ of $G$ is defined as the spectrum of $A(G)$. Since $A$ is symmetric, its eigenvalues $\lambda_{1}(G), \lambda_{2}(G), \ldots, \lambda_{n}(G)$ are real, and we assume that $\lambda_{1}(G) \geq \lambda_{2}(G) \geq \cdots \geq \lambda_{n}(G)$. We call $\rho(G)=\lambda_{1}(G)$ the adjacency spectral radius of $G$.

Let $G^{\sigma}$ be a simple graph with an orientation $\sigma$, which assigns to each edge of $G$ a direction so that $G^{\sigma}$ becomes a directed graph. The skew-adjacency matrix $S=S\left(G^{\sigma}\right)$ is the real skew-symmetric matrix $\left[s_{j k}\right]_{n \times n}$, where $s_{j k}=1$ and $s_{k j}=-1$ if $\left(v_{j}, v_{k}\right)$ is an arc of $G^{\sigma}$, otherwise $s_{j k}=s_{k j}=0$. We call $\operatorname{det}(\lambda I-S)$ the skew-characteristic polynomial of $G^{\sigma}$, denoted by $\phi\left(G^{\sigma} ; \lambda\right)$. The skew spectrum $S_{p}\left(G^{\sigma}\right)$ of $G^{\sigma}$ is defined as the spectrum of $S\left(G^{\sigma}\right)$. Since $S$ is skew-symmetric, its eigenvalues are purely imaginary numbers $\lambda_{1}\left(G^{\sigma}\right) i, \lambda_{2}\left(G^{\sigma}\right) i, \ldots, \lambda_{n}\left(G^{\sigma}\right) i$. Also, we assume that $\lambda_{1}\left(G^{\sigma}\right) \geq \lambda_{2}\left(G^{\sigma}\right) \geq \cdots \geq \lambda_{n}\left(G^{\sigma}\right)$ and call $\varrho\left(G^{\sigma}\right)=\lambda_{1}\left(G^{\sigma}\right)$ the skew-spectral radius of $G^{\sigma}$.

In this paper, we will denote by $\mathfrak{D}(G)$ the set of all the oriented graphs obtained from $G$ by giving an arbitrary orientation to each edge. Also, we refer to [1-3] for more terminologies and notations not defined here.

Unlike the adjacency matrix of a graph, there is little research on the skew-adjacency matrix $S\left(G^{\sigma}\right)$, except that into enumeration of perfect matchings of a graph. As early as in 1947, Tutte [4] derived his famous characterization of the graphs with no perfect match-

(C) 2012 Xu; licensee Springer. This is an Open Access article distributed under the terms of the Creative Commons Attribution License (http://creativecommons.org/licenses/by/2.0), which permits unrestricted use, distribution, and reproduction in any medium, provided the original work is properly cited. 
ings. Tutte's result motivates a lot of work on the matchings polynomial and enumerating perfect matchings of graphs in terms of its skew-adjacency matrix; see, for example, [5-7] and references therein.

Recently, many researchers have paid a great deal of attention to the spectral properties of skew-symmetric matrices in terms of oriented graphs. IMA-ISU research group on minimum rank [8] studied the minimum rank of skew-symmetric matrices. In 2009, Shader and So [9] investigated the spectra of the skew-adjacency matrix of an oriented graph. And in 2010, Adiga et al. [10] discussed the properties of the skew energy of an oriented graph. In the papers [11] and [12], all the coefficients of the skew-characteristic polynomial of $G^{\sigma}$ in terms of $G$ were interpreted.

The motivation of this paper is to study more carefully the skew spectra of oriented graphs. In Section 2, we firstly establish a relation between $\varrho\left(G^{\sigma}\right)$ and $\rho(G)$. In Section 3, we give some results on the skew-spectral radii of $G^{\sigma}$ and its oriented subgraphs. Finally, in Section 4, we give an application of the previous results - to obtain a sharp upper bound of the skew-spectral radius of an oriented unicyclic graph.

\section{A relation between $\rho(G)$ and $\varrho\left(G^{\sigma}\right)$}

It is interesting to discuss the relations between $\rho(G)$ and $\varrho\left(G^{\sigma}\right)$. Let $G$ be a simple graph. We denoted by $N(v)$ the neighborhood of the vertex $v$ in $G$, by $G-e$ the subgraph obtained from $G$ by deleting the edge $e$ and by $G-v$ the subgraph obtained from $G$ by removing the vertex $v$ together with all edges incident to it. A walk $W$ of length $k$ from $u$ to $v$ in $G$ is a sequence of $k+1$ vertices starting at $u$ and ending at $v$ such that consecutive vertices are adjacent. If all vertices in a walk are distinct, then such a walk is called a path of $G$, denoted by $P$. Let $P=v_{1} v_{2} \cdots v_{k}$ be a path with $k \geq 3$. Then $P$ together with the edge $v_{k} v_{1}$ is called a cycle of $G$, denoted by $C$.

Let $G^{\sigma} \in \mathfrak{D}(G)$ and $S\left(G^{\sigma}\right)$ be its skew-adjacency matrix. Let $W=u_{1} u_{2} \cdots u_{k} u_{k+1}$ be a walk of $G$ (we often call it a $(k+1)$-walk). The sign of $W$ in $G^{\sigma}$, denoted by $\operatorname{sgn}\left(W^{\sigma}\right)$, is defined by

$$
s_{12} s_{23} \cdots s_{k-1, k} s_{k, k+1} \text {. }
$$

Let $\bar{W}=u_{k+1} u_{k} \cdots u_{2} u_{1}$ be the walk by inverting the order of the vertices along the walk $W$. Then one can find that

$$
\operatorname{sgn}\left(\bar{W}^{\sigma}\right)= \begin{cases}-\operatorname{sgn}\left(W^{\sigma}\right), & \text { if } k \text { is odd } \\ \operatorname{sgn}\left(W^{\sigma}\right), & \text { if } k \text { is even }\end{cases}
$$

Obviously, for an even closed walk (that is to say, $u_{k+1}=u_{1}$ ), we can simply refer to it as a positive (or negative) even closed walk according to its sign, regardless of the order of its vertices. Similarly, we can define a positive (or negative) even cycle. Moreover, an even cycle $C_{2 k}$ with $2 k$ vertices is said to be oriented uniformly if its $\operatorname{sign}$ in $G^{\sigma}$ is $\operatorname{sgn}\left(C_{2 k}^{\sigma}\right)=(-1)^{k}$.

Note that in terms of defining walks, paths, cycles etc., we focus only on the underlying undirected graph. And their signs are based on the oriented graph.

For $G^{\sigma} \in \mathfrak{D}(G)$, one reverses the orientations of all the arcs incident to a particular vertex of $G^{\sigma}$. We call such an operation a reversal of $G^{\sigma}$. Let $G^{\tau}$ be the digraph obtained from $G^{\sigma}$ 
by doing some reversals. Then $G^{\sigma}$ and $G^{\tau}$ are said to be quasi-isomorphic and denoted by $G^{\sigma} \simeq G^{\tau}$. For example, if $T$ is a tree and $T^{\sigma}, T^{\tau} \in \mathfrak{D}(T)$, then we always have $T^{\sigma} \simeq T^{\tau}$.

On quasi-isomorphic oriented graphs, Adiga et al. [10] obtained the following result.

Lemma 2.1 ([10]) Let $G^{\sigma} \in \mathfrak{D}(G)$ and $G^{\tau} \simeq G^{\sigma}$. Then the skew-adjacency matrices $S\left(G^{\sigma}\right)$ and $S\left(G^{\tau}\right)$ are orthogonally similar. And therefore,

$$
S_{p}\left(G^{\sigma}\right)=S_{p}\left(G^{\tau}\right)
$$

For oriented bipartite graphs, we have

Lemma 2.2 Let $G=(U, V ; E)$ be a bipartite graph and $|U|=m,|V|=n$. The adjacency matrix of $G$ is

$$
A(G)=\left(\begin{array}{cc}
0 & X \\
X^{T} & 0
\end{array}\right) .
$$

Let $G^{\sigma} \in \mathfrak{D}(G)$ and each even cycle of $G$ be oriented uniformly. Then $G^{\sigma} \simeq D$, where $D \in$ $\mathfrak{D}(G)$ and its skew-adjacency matrix is

$$
S(D)=\left(\begin{array}{cc}
0 & X \\
-X^{T} & 0
\end{array}\right) .
$$

Proof Firstly, we will prove that each even closed walk of $G$ is oriented uniformly in $G^{\sigma}$ too. That is to say, for an arbitrary closed $2 k$-walk of $G$, its sign in $G^{\sigma}$ is $(-1)^{k}$. Let $W=u_{1} u_{2} \cdots u_{2 k} u_{1}$ be a closed $2 k$-walk of $G$. We may assume the closed $2 k$-walk is exactly constituted by the cycles (or closed 2 -walks) $C_{j}$ with $2 k_{j}$ vertices $(j=1,2, \ldots, r)$. Hence,

$$
\operatorname{sgn}(W)=(-1)^{k_{1}+k_{2}+\cdots+k_{r}}=(-1)^{k} .
$$

Now, to prove the lemma, we can consider the following two cases.

Case 1. $G \cong K_{m n}$;

Let $u_{1} \in U, v_{1} \in V$. Obviously, we can take some reversals to obtain a new oriented graph $G^{\tau}$ such that all the $\operatorname{arcs}\left(u_{1}, v_{j}\right),\left(u_{l}, v_{1}\right) \in G^{\tau}(j=1,2, \ldots, n, l=1,2, \ldots, m)$. Now, if $G^{\tau} \not D$, then there exists an $\operatorname{arc}\left(v_{p}, u_{q}\right) \in G^{\tau}$, where $u_{q} \in U$ and $v_{p} \in V$. Thus, for the cycle $C_{4}=$ $u_{1} v_{1} u_{q} v_{p} u_{1}$, we have $\operatorname{sgn}\left(C_{4}^{\sigma}\right)=\operatorname{sgn}\left(C_{4}^{\tau}\right)=-1 \neq(-1)^{2}$. Contradicting the condition of this lemma that each even cycle of $G$ is oriented uniformly in $G^{\sigma}, G^{\tau} \cong D$, and then $G^{\sigma} \simeq D$.

Case 2. $G \not K_{m n}$;

Without loss of generality, we may assume $G$ is connected. Then there must exist two vertices $u \in U, v \in V$ not adjacent in $G$. Let $W_{1}=u u_{1} \cdots u_{2 k_{1}} v$ be a walk of $G$. Now, we join the vertices $u$ and $v$ and suppose the resulting graph is $G_{1}$. Obviously, we can give the edge $u v$ a direction (for instance, from $u$ to $v$ ) such that the closed $2\left(k_{1}+1\right)$-walk $W=W_{1}+u v$ satisfies $\operatorname{sgn}\left(W^{\varsigma}\right)=(-1)^{k_{1}+1}$ in $G_{1}^{\varsigma}=G^{\sigma}+(u, v)$. Of course, it means that

$$
\operatorname{sgn}\left(W_{1}^{\sigma}\right)=\operatorname{sgn}\left(W_{1}^{\zeta}\right)=(-1)^{k_{1}} .
$$


If there exists another walk $W_{2}=v v_{1} \cdots v_{2 k_{2}} u$ in $G$, then

$$
W_{0}=u u_{1} \cdots u_{2 k_{1}} v v_{1} \cdots v_{2 k_{2}} u
$$

is a closed $2\left(k_{1}+k_{2}+1\right)$-walk of $G$. Thus,

$$
\operatorname{sgn}\left(W_{2}^{\sigma}\right)=\frac{(-1)^{k_{1}+k_{2}+1}}{(-1)^{k_{1}}}=(-1)^{k_{2}+1}
$$

Hence, the closed $2\left(k_{2}+1\right)$-walk $\bar{W}=u v+W_{2}$ of $G_{1}$ satisfies $\operatorname{sgn}\left(\bar{W}^{\zeta}\right)=(-1)^{k_{2}+1}$ in $G_{1}^{\varsigma}$. Thus, each even cycle of $G_{1}$ is oriented uniformly in $G_{1}^{\varsigma}$. It tells us that the graph $G_{1}$ satisfies the conditions of this lemma too. Furthermore, if the result holds for $G_{1}$, we can get immediately the same result for the graph $G$. By taking some similar operations, we can obtain the graph $K_{m n}$. And then, by the Case 1 and the above discussion, we know the result holds.

Moreover, the following result tells us that $S_{p}(D)=i S_{p}(G)$ for a bipartite graph $G$ and its oriented graph $D$ defined as in Lemma 2.2 .

Lemma 2.3 ([9]) Let

$$
A=\left(\begin{array}{cc}
0 & X \\
X^{T} & 0
\end{array}\right)
$$

and

$$
B=\left(\begin{array}{cc}
0 & X \\
-X^{T} & 0
\end{array}\right)
$$

be two real matrices. $S_{p}(A)$ and $S_{p}(B)$ are denoted by the spectrum of $A$ and $B$ respectively. Then $S_{p}(B)=i S_{p}(A)$.

To obtain the main result of this section, we also need the following result.

Lemma 2.4 ([2]) Let $A$ be an irreducible nonnegative $n \times n$ matrix and $B$ be a complex $n \times n$ matrix such that $|B| \leq A$ (entry-wise). Then $|\lambda(B)| \leq \rho(A)$ for each eigenvalue $\lambda(B)$ of $B$; and $\lambda(B)=\rho(A) e^{i \theta}$ iff $B=e^{i \theta} L A L^{-1}$, where $e^{i \theta}=\cos \theta+i \sin \theta$ and $|L|=I_{n}$, the identity matrix.

Now, by the above lemmas, we can give the relation between $\rho(G)$ and $\varrho\left(G^{\sigma}\right)$ as follows.

Theorem 2.1 Let $G$ be a connected graph, $G^{\sigma} \in \mathfrak{D}(G)$. Then $\varrho\left(G^{\sigma}\right) \leq \rho(G)$ with equality if and only if $G$ is a bipartite graph and each even cycle of $G$ is oriented uniformly in $G^{\sigma}$.

Proof By Lemma 2.4, we know that $\varrho\left(G^{\sigma}\right) \leq \rho(G)$.

Now suppose that $\varrho\left(G^{\sigma}\right)=\rho(G)$. By Lemma 2.4, we have $S\left(G^{\sigma}\right)= \pm i L A(G) L^{-1}$, where $|L|=I_{n}$, the identity matrix. Let $S\left(G^{\sigma}\right)=\left[s_{j l}\right], A(G)=\left[a_{j l}\right]$ and $L=\operatorname{diag}\left(d_{j}\right)$. Then it is not 
difficult to know that

$$
s_{j l}= \pm i a_{j l} \frac{d_{j}}{d_{l}} .
$$

Let $C=u_{1} u_{2} \cdots u_{r} u_{1}$ be a $r$-cycle of $G$. Then $\operatorname{sgn}\left(C^{\sigma}\right)=s_{12} s_{23} \cdots s_{r-1, r} s_{r 1}$. Thus,

$$
\operatorname{sgn}\left(C^{\sigma}\right)=\frac{d_{1}}{d_{2}} \frac{d_{2}}{d_{3}} \cdots \frac{d_{r-1}}{d_{r}} \frac{d_{r}}{d_{1}}( \pm i)^{r}=( \pm i)^{r}
$$

Since $\operatorname{sgn}\left(C^{\sigma}\right)=1$ or $-1, r$ must be an even number. Hence, $G$ is a bipartite graph, and each even cycle of $G$ is oriented uniformly in $G^{\sigma}$.

Conversely, let $G$ be a bipartite graph and each even cycle of $G$ be oriented uniformly in $G^{\sigma}$. Assume the adjacency matrix of $G$ is

$$
A(G)=\left(\begin{array}{cc}
0 & X \\
X^{T} & 0
\end{array}\right) .
$$

Then by Lemma $2.2, G^{\sigma} \simeq D$ and the skew-adjacency matrix of $D$ is

$$
S(D)=\left(\begin{array}{cc}
0 & X \\
-X^{T} & 0
\end{array}\right)
$$

Thus, by Lemma 2.1 and Lemma 2.3, we have $\varrho\left(G^{\sigma}\right)=\varrho(D)=\rho(G)$.

Remark 2.1 By this theorem and those known results on the adjacency spectral radii of bipartite graphs, we can obtain similar results on the skew-spectral radii of oriented bipartite graphs.

For example, we can give the following sharp upper bound on the skew-spectral radii of oriented bipartite graphs.

Theorem 2.2 Let $G=(U, V ; E)$ be a bipartite graph with $|U|=n_{1}$ and $|V|=n_{2}$. Let $G^{\sigma} \in$ $\mathfrak{D}(G)$. Then

$$
\varrho\left(G^{\sigma}\right) \leq \sqrt{n_{1} n_{2}}
$$

with equality if and only if $G^{\sigma} \simeq D$, where $D \in \mathfrak{D}\left(K_{n_{1} n_{2}}\right)$, and its skew-adjacency matrix is

$$
S(D)=\left(\begin{array}{cc}
0 & J_{n_{1} n_{2}} \\
-J_{n_{1} n_{2}}^{T} & 0
\end{array}\right),
$$

where $J_{n_{1} n_{2}}$ is the $n_{1} \times n_{2}$ matrix with all entries equal to 1 .

Proof It is well known that $\rho(G) \leq \sqrt{n_{1} n_{2}}$ with equality if and only if $G \cong K_{n_{1} n_{2}}$. Thus, by Lemma 2.2 and Theorem 2.1, we know the result holds. 


\section{Some relations on the skew-spectral radii of an oriented graph and its subgraphs}

In this section, we will discuss the relations on the skew-spectral radii of an oriented graph and its subgraphs due to vertex (or edge) deletion. Certainly, in this paper, each subgraph of an oriented graph is also referred to as an oriented graph and preserves the direction of each arc, even if we do not indicate specially.

Let $G$ be a simple graph and $G^{\sigma} \in \mathfrak{D}(G)$. A subgraph $H$ of $G$ is called a basic subgraph if each component of $H$ is an edge or an even cycle. Of course, $|V(H)|$ is an even number.

In the papers [11] and [12], the following result was obtained.

Lemma 3.1 ([11, 12]) Let $G^{\sigma} \in \mathfrak{D}(G)$ and the skew-characteristic polynomial is

$$
\phi\left(G^{\sigma}, \lambda\right)=\sum_{j=0}^{n} a_{j} \lambda^{n-j}=\lambda^{n}+a_{1} \lambda^{n-1}+a_{2} \lambda^{n-2}+\cdots+a_{n-1} \lambda+a_{n}
$$

Then the coefficient of $\lambda^{n-j}$ satisfies

$$
a_{j}= \begin{cases}0, & \text { if } j=2 r+1 ; \\ \sum_{H}(-1)^{c^{+}} 2^{c}, & \text { if } j=2 r,\end{cases}
$$

where the summation is over all the basic subgraphs of $G$ having $2 r$ vertices $\left(0 \leq r \leq\left\lfloor\frac{n}{2}\right\rfloor\right)$, $c^{+}$and $c$ are respectively the number of positive even cycles and the number of even cycles contained in $H^{\sigma}$.

As an application of this lemma, we can obtain the following results (the result (b) of the following theorem was also obtained by Hou and Lei [12]) which can be used to find recursions for the skew-characteristic polynomial of some oriented graphs.

Theorem 3.1 Let $G=(V, E)$ be a simple graph, $u \in V(G), e \in E(G)$. Then the skewcharacteristic polynomial of an oriented graph $G^{\sigma}$ satisfies the following identities:

(a) $\phi\left(G^{\sigma}, \lambda\right)=\lambda \phi\left(G^{\sigma}-u, \lambda\right)+\sum_{v} \phi\left(G^{\sigma}-u-v, \lambda\right)+2 \sum_{Z}(-1)^{\delta(Z)} \phi\left(G^{\sigma}-V(Z), \lambda\right)$,

where the first summation is over all the vertices in $N(u)$ and the second summation is over all the even cycles of $G$ containing the vertex $u$. Moreover,

$\delta(Z)= \begin{cases}1, & \text { if } Z \text { is a positive even cycle in } G^{\sigma} \\ 0, & \text { if } Z \text { is a negative even cycle in } G^{\sigma}\end{cases}$

(b) $\phi\left(G^{\sigma}, \lambda\right)=\phi\left(G^{\sigma}-e, \lambda\right)+\phi\left(G^{\sigma}-u-v, \lambda\right)+2 \sum_{Z}(-1)^{\delta(Z)} \phi\left(G^{\sigma}-V(Z), \lambda\right)$,

where $e=u v$ and the summation is over all the even cycles of $G$ containing the edge e.

Proof (a) Obviously, all basic subgraphs of $G$ having $j=2 r$ vertices can be divided into three parts: those that do not contain the vertex $u$, those that contain $u$ together with a 
neighbor $v$ as an edge of it, and those that contain $u$ together with the vertices of an even cycle containing $u$ as the component of it. Then $a_{j}=a_{j}^{(1)}+a_{j}^{(2)}+a_{j}^{(3)}$, where

$$
a_{j}^{(1)}=\sum_{H_{1}}(-1)^{c^{+}} 2^{c}
$$

the summation is over all the basic subgraphs of $G-u$ having $j=2 r$ vertices;

$$
a_{j}^{(2)}=\sum_{v} a_{j-2}(v)
$$

the summation is over all the vertices in $N(u)$. And $a_{j-2}(v)=\sum_{H_{2}}(-1)^{c^{+}} 2^{c}$, where the summation is over all the basic subgraphs of $G-u-v$ having $j-2$ vertices;

$$
a_{j}^{(3)}=2 \sum_{Z}(-1)^{\delta(Z)} a_{j-|V(Z)|}
$$

the summation is over all the even cycles of $G$ containing the vertex $u$. And $a_{j-|V(Z)|}=$ $\sum_{H_{3}}(-1)^{c^{+}} 2^{c}$, where the summation is over all the basic subgraphs of $G-V(Z)$ having $j-|V(Z)|$ vertices.

When $j$ is an odd number, we write $a_{j}^{(t)}=a_{j}=0(t=1,2,3)$.

Thus,

$$
\begin{aligned}
& \sum_{j=0}^{n} a_{j}^{(1)} \lambda^{n-j}=\lambda \sum_{j=0}^{n-1} a_{j}^{(1)} \lambda^{n-1-j}=\lambda \phi\left(G^{\sigma}-u, \lambda\right), \\
& \sum_{j=0}^{n} a_{j-2}(v) \lambda^{n-j}=\sum_{j=2}^{n} a_{j-2}(v) \lambda^{n-j}=\phi\left(G^{\sigma}-u-v, \lambda\right), \\
& \sum_{j=0}^{n} a_{j-|V(Z)|} \lambda^{n-j}=\sum_{j=|V(Z)|}^{n} a_{j-|V(Z)|} \lambda^{n-j}=\phi\left(G^{\sigma}-V(Z), \lambda\right) .
\end{aligned}
$$

Thus, the result holds.

(b) The proof is similar to that of (a).

Now, we consider the relations on the skew-spectral radii of an oriented graph and its subgraphs due to vertex (or edge) deletion. Firstly, according to a classical result in the matrix theory, we have

Lemma 3.2 (Interlacing of eigenvalues) Let $G$ be a simple graph on $n$ vertices, $v \in V(G)$, $G^{\sigma} \in \mathfrak{D}(G)$. Suppose

$$
\begin{aligned}
& S_{p}\left(G^{\sigma}\right)=\left\{\lambda_{1} i, \lambda_{2} i, \ldots, \lambda_{n} i\right\}, \\
& S_{p}\left(G^{\sigma}-v\right)=\left\{\mu_{1} i, \mu_{2} i, \ldots, \mu_{n-1} i\right\} .
\end{aligned}
$$

Then we have

$$
\lambda_{1} \geq \mu_{1} \geq \lambda_{2} \geq \mu_{2} \geq \cdots \geq \lambda_{n-1} \geq \mu_{n-1} \geq \lambda_{n}
$$


Proof Let $S\left(G^{\sigma}\right)$ be the skew-adjacency matrix of $G^{\sigma}$. Since $i S\left(G^{\sigma}\right)$ is a Hermitian matrix, by the well-known Cauchy-Poincare theorem, we know the result holds.

Now, we can get the following strict inequalities on the skew-spectral radii of an oriented graph and its subgraphs.

Theorem 3.2 Let $G$ be a connected simple graph on $n$ vertices, $G^{\sigma} \in \mathfrak{D}(G)$. If each even cycle of $G$ is oriented uniformly in $G^{\sigma}$, then

(a) $\varrho\left(G^{\sigma}\right)>\varrho\left(G^{\sigma}-u\right)$, where $u$ is an arbitrary vertex of $G$.

(b) $\varrho\left(G^{\sigma}\right)>\varrho\left(G^{\sigma}-e\right)$, where $e$ is an arbitrary edge of $G$. Moreover, for $\varrho \geq \varrho\left(G^{\sigma}-e\right)$, we have $i^{-n} \phi\left(G^{\sigma}-e, \varrho i\right)>i^{-n} \phi\left(G^{\sigma}, \varrho i\right)$.

Proof (a) We prove the statement by induction on $n$. Obviously, the result holds for $n=$ 2,3 .

Now, suppose the result holds when the number of vertices is smaller than $n$. We consider the case that the number of vertices equals $n$. Firstly, we have

$$
\phi\left(G^{\sigma}, \lambda\right)=\lambda \phi\left(G^{\sigma}-u, \lambda\right)+\sum_{v} \phi\left(G^{\sigma}-u-v, \lambda\right)+2 \sum_{Z}(-1)^{\delta(Z)} \phi\left(G^{\sigma}-V(Z), \lambda\right) .
$$

Let $G-u=\bigcup_{l=1}^{r} G_{l}$ and $\varrho\left(G^{\sigma}-u\right)=\varrho\left(G_{1}^{\sigma}\right)=\lambda_{0}$.

Case 1. $\left|V\left(G_{1}\right)\right| \leq n-2$;

Then, by Lemma 3.2, $\varrho\left(G^{\sigma}\right) \geq \varrho\left(\left(G_{1} \circ u\right)^{\sigma}\right)$, where $G_{1} \circ u$ is the subgraph of $G$ induced by the vertex set $V\left(G_{1}\right) \cup u$. By induction assumption, we have

$$
\varrho\left(\left(G_{1} \circ u\right)^{\sigma}\right)>\varrho\left(G_{1}^{\sigma}\right)=\varrho\left(G^{\sigma}-u\right)
$$

And then

$$
\varrho\left(G^{\sigma}\right)>\varrho\left(G^{\sigma}-u\right)
$$

Case 2. $\left|V\left(G_{1}\right)\right|=n-1$;

Then $G_{1} \cong G-u$ and is connected. By induction assumption, we know $\varrho\left(G^{\sigma}-u-v\right)<\lambda_{0}$ for each vertex $v$ in $N(u)$. Thus,

$$
\phi\left(G^{\sigma}, \lambda_{0} i\right)=\sum_{v} \phi\left(G^{\sigma}-u-v, \lambda_{0} i\right)+2 \sum_{Z}(-1)^{\delta(Z)} \phi\left(G^{\sigma}-V(Z), \lambda_{0} i\right) .
$$

Obviously, for each vertex $v \in N(u)$, we have

$$
\phi\left(G^{\sigma}-u-v, \lambda_{0} i\right)=i^{n-2} q_{v}, \quad q_{v}>0 .
$$

On the other hand, for an arbitrary $2 k$-cycle $Z$ (if there exists) containing $u$, we have

$$
(-1)^{\delta(Z)}= \begin{cases}-1, & \text { if } k \text { is even } \\ 1, & \text { if } k \text { is odd }\end{cases}
$$


It means that

$$
(-1)^{\delta(Z)}=(-1)^{k-1}=i^{2 k-2} .
$$

And thus,

$$
(-1)^{\delta(Z)} \phi\left(G^{\sigma}-V(Z), \lambda_{0} i\right)=i^{n-2} q_{Z}, \quad q_{Z}>0 .
$$

Hence,

$$
\phi\left(G^{\sigma}, \lambda_{0} i\right)=i^{n-2} q
$$

where $q>0$. Thus the result holds.

(b) Since

$$
\phi\left(G^{\sigma}, \lambda\right)=\phi\left(G^{\sigma}-e, \lambda\right)+\phi\left(G^{\sigma}-u-v, \lambda\right)+2 \sum_{Z}(-1)^{\delta(Z)} \phi\left(G^{\sigma}-V(Z), \lambda\right) .
$$

By the result (a), we have

$$
\varrho\left(G^{\sigma}-u-v\right)=\varrho\left(\left(G^{\sigma}-e\right)-u-v\right)<\varrho\left(G^{\sigma}-e\right) .
$$

And, for an arbitrary even cycle $Z$ (if it exists) containing $e$, we have

$$
\varrho\left(G^{\sigma}-V(Z)\right)<\varrho\left(G^{\sigma}-e\right)
$$

Then, for $\varrho \geq \varrho\left(G^{\sigma}-e\right)$, we have

$$
\phi\left(G^{\sigma}-e, \varrho i\right)-\phi\left(G^{\sigma}, \varrho i\right)=i^{n} q
$$

where $q>0$. Thus,

$$
i^{-n} \phi\left(G^{\sigma}-e, \varrho i\right)>i^{-n} \phi\left(G^{\sigma}, \varrho i\right) .
$$

Furthermore, we know $\varrho\left(G^{\sigma}\right)>\varrho\left(G^{\sigma}-e\right)$.

Moreover, by this theorem, we have the following

Corollary 3.1 Let $G$ be a connected simple graph of order $n, G^{\sigma} \in \mathfrak{D}(G), V^{\prime} \subset V(G), E^{\prime} \subset$ $E(G)$. If each even cycle of $G$ is oriented uniformly in $G^{\sigma}$, then

(a) $\varrho\left(G^{\sigma}\right)>\varrho\left(G^{\sigma}-V^{\prime}\right)$.

(b) $i^{-n} \phi\left(G^{\sigma}-E^{\prime}, \varrho i\right)>i^{-n} \phi\left(G^{\sigma}, \varrho i\right)$ for $\varrho \geq \varrho\left(G^{\sigma}-E^{\prime}\right)$. In particular, we have $\varrho\left(G^{\sigma}\right)>\varrho\left(G^{\sigma}-E^{\prime}\right)$

Remark 3.1 Note that the above results will help us to compare the skew-spectral radii of two oriented graphs and then to clarify the skew-spectral properties of some oriented graphic classes. In the next section, we will just give such an example. 


\section{An application of the previous results}

A so-called unicyclic graph is a connected simple graph in which the number of edges equals the number of vertices. In this section, we will give an application of the previous results - to obtain a sharp upper bound of the skew-spectral radius of an oriented unicyclic graph.

For convenience, we write

$$
\begin{aligned}
& \mathcal{U}_{n}=\{G \mid G \text { is a unicyclic graph with } n \text { vertices }\} \\
& \mathcal{U}_{n}(k)=\left\{G \mid G \text { is a unicyclic graph in } \mathcal{U}_{n} \text { containing the cycle } C_{k}\right\}
\end{aligned}
$$

Also, we denoted by $C_{k}^{n-k}$ the unicyclic graph obtained from $C_{k}$ by joining a vertex of $C_{k}$ with $(n-k) K_{1}$.

Regarding the adjacency spectral radii of unicyclic graphs, Hong [13] obtained the following results.

Lemma 4.1 ([13]) Let $G \in \mathcal{U}_{n}(k)$. Then

$$
\rho(G) \leq \rho\left(C_{k}^{n-k}\right)
$$

with equality if and only if $G \cong C_{k}^{n-k}$.

Lemma $4.2([13]) \rho\left(C_{k}^{n-k}\right)>\rho\left(C_{k+1}^{n-k-1}\right), 3 \leq k \leq n-1$.

Now, we begin to discuss the skew-spectral radii of unicyclic graphs. Firstly, we consider the bipartite unicyclic graphs. For this case, we have

Lemma 4.3 Let $G \in \mathcal{U}_{n}(2 k)$ and $G^{\sigma} \in \mathfrak{D}(G)$. Then

$$
\varrho\left(G^{\sigma}\right) \leq \rho\left(C_{4}^{n-4}\right)=\sqrt{\frac{n+\sqrt{n^{2}-8 n+32}}{2}} .
$$

The equality holds if and only if $G \cong C_{4}^{n-4}$ and the sign of the cycle in $G^{\sigma}$ is positive.

Proof By Lemma 2.1, we know $\varrho\left(G^{\sigma}\right) \leq \rho(G)$ and the equality holds if and only if the sign of the $2 k$-cycle in $G^{\sigma}$ is $(-1)^{k}$. Moreover, by Lemmas 4.1 and $4.2, \rho(G) \leq \rho\left(C_{4}^{n-4}\right)$ and the equality holds if and only if $G \cong C_{4}^{n-4}$.

Thus, $\varrho\left(G^{\sigma}\right) \leq \rho\left(C_{4}^{n-4}\right)$ with equality if and only if $G \cong C_{4}^{n-4}$ and the sign of the cycle in $G^{\sigma}$ is $(-1)^{2}=1$. That is to say the sign of the cycle in $G^{\sigma}$ is positive.

For the graph $C_{4}^{n-4}$, it is not difficult to know that its characteristic polynomial is

$$
P\left(C_{4}^{n-4}, \lambda\right)=\lambda^{n-4}\left[\lambda^{4}-n \lambda^{2}+2(n-4)\right] .
$$

And then

$$
\rho\left(C_{4}^{n-4}\right)=\sqrt{\frac{n+\sqrt{n^{2}-8 n+32}}{2}} .
$$

This completes the proof. 
Now, we begin to consider the non-bipartite unicyclic graphs. Let $G \in \mathcal{U}_{n}(2 k+1)$ and $G^{\sigma}, G^{\tau} \in \mathfrak{D}(G)$. We can easily know that $G^{\sigma} \simeq G^{\tau}$ and $S_{p}\left(G^{\sigma}\right)=S_{p}\left(G^{\tau}\right)$. Moreover, we have

Lemma 4.4 Let $G \in \mathcal{U}_{n}(2 k+1)$ and $G^{\sigma} \in \mathfrak{D}(G)$. Then $i^{-n} \phi\left(G^{\sigma}, \varrho i\right) \geq i^{-n} \phi\left(D_{2 k+1}^{n-2 k-1}, \varrho i\right)$ for $\varrho \geq \varrho\left(G^{\sigma}\right)$. And thus

$$
\varrho\left(G^{\sigma}\right) \leq \varrho\left(D_{2 k+1}^{n-2 k-1}\right)
$$

where $D_{2 k+1}^{n-2 k-1} \in \mathfrak{D}\left(C_{2 k+1}^{n-2 k-1}\right)$. The above two equalities hold if and only if $G \cong C_{2 k+1}^{n-2 k-1}$.

Proof We prove the statement by induction on $n-2 k-1$. Obviously, the result holds for $n-2 k-1=0,1$.

Now, suppose the result holds for $n-2 k-1<t$. We consider the case that $n-2 k-1=t$. Assume $G \in \mathcal{U}_{n}(2 k+1)$ and $G ¥ C_{2 k+1}^{n-2 k-1}$. Let $u \in V(G)$ and $d(u)=1, u v \in E(G)$, then

$$
\begin{aligned}
& \phi\left(G^{\sigma}, \lambda\right)=\lambda \phi\left(G^{\sigma}-u, \lambda\right)+\phi\left(G^{\sigma}-u-v, \lambda\right), \\
& \phi\left(D_{2 k+1}^{n-2 k-1}, \lambda\right)=\lambda \phi\left(D_{2 k+1}^{n-2 k-2}, \lambda\right)+\phi\left(D_{0}, \lambda\right),
\end{aligned}
$$

where $D_{0} \in \mathfrak{D}\left(P_{2 k} \cup(n-2 k-2) K_{1}\right)$. By induction hypothesis, for $\varrho \geq \varrho\left(G^{\sigma}\right)>\varrho\left(G^{\sigma}-u\right)$, we have

$$
\begin{aligned}
& i^{-(n-1)} \phi\left(G^{\sigma}-u, \varrho i\right)>i^{-(n-1)} \phi\left(D_{2 k+1}^{n-2 k-2}, \varrho i\right), \\
& \varrho\left(G^{\sigma}-u\right)<\varrho\left(D_{2 k+1}^{n-2 k-2}\right) .
\end{aligned}
$$

Since $P_{2 k} \cup(n-2 k-2) K_{1}$ is a proper spanning subgraph of $G-u-v$, by Corollary 3.1, for $\varrho>\varrho\left(G^{\sigma}-u\right)>\varrho\left(G^{\sigma}-u-v\right)$, we have

$$
i^{-(n-2)} \phi\left(G^{\sigma}-u-v, \varrho i\right)<i^{-(n-2)} \phi\left(D_{0}, \varrho i\right) .
$$

So, for $\varrho \geq \varrho\left(G^{\sigma}\right)$, we have

$$
\begin{aligned}
& i^{-n} \phi\left(G^{\sigma}, \varrho i\right)-i^{-n} \phi\left(D_{2 k+1}^{n-2 k-1}, \varrho i\right) \\
& =\varrho i^{-(n-1)}\left[\phi\left(G^{\sigma}-u, \varrho i\right)-\phi\left(D_{2 k+1}^{n-2 k-2}, \varrho i\right)\right] \\
& \quad+i^{-(n-2)}\left[\phi\left(D_{0}, \varrho i\right)-\phi\left(G^{\sigma}-u-v, \varrho i\right)\right]>0 .
\end{aligned}
$$

Hence, the result follows.

Furthermore, we have

Lemma 4.5 Let $D_{2 k+1}^{n-2 k-1} \in \mathfrak{D}\left(C_{2 k+1}^{n-2 k-1}\right)$ and $D_{2 k-1}^{n-2 k+1} \in \mathfrak{D}\left(C_{2 k-1}^{n-2 k+1}\right)(n>5, k \geq 2)$. Then for $\varrho \geq \varrho\left(D_{2 k+1}^{n-2 k-1}\right)$, we have

$$
i^{-n} \phi\left(D_{2 k+1}^{n-2 k-1}, \varrho i\right)>i^{-n} \phi\left(D_{2 k-1}^{n-2 k+1}, \varrho i\right) .
$$

In particular, $\varrho\left(D_{2 k+1}^{n-2 k-1}\right)<\varrho\left(D_{2 k-1}^{n-2 k+1}\right)$. 
Proof We prove the result by induction on $n$. Obviously, the result holds for $n=6$.

Now, suppose the result holds for the number of vertices less than $n$. We consider the case that the number of vertices is $n$. By Theorem 3.1, we know

$$
\begin{aligned}
& \phi\left(D_{2 k+1}^{n-2 k-1}, \lambda\right)=\lambda \phi\left(D_{2 k+1}^{n-2 k-2}, \lambda\right)+\phi\left(D_{1}, \lambda\right), \\
& \phi\left(D_{2 k-1}^{n-2 k+1}, \lambda\right)=\lambda \phi\left(D_{2 k-1}^{n-2 k}, \lambda\right)+\phi\left(D_{2}, \lambda\right),
\end{aligned}
$$

where $D_{1} \in \mathfrak{D}\left(P_{2 k} \cup(n-2 k-2) K_{1}\right), D_{2} \in \mathfrak{D}\left(P_{2 k-2} \cup(n-2 k) K_{1}\right)$. By induction hypothesis and Corollary 3.1, for $\varrho \geq \varrho\left(D_{2 k+1}^{n-2 k-1}\right)$, we have

$$
\begin{aligned}
& i^{-(n-1)} \phi\left(D_{2 k+1}^{n-2 k-2}, \varrho i\right)>i^{-(n-1)} \phi\left(D_{2 k-1}^{n-2 k}, \varrho i\right), \\
& i^{-(n-2)} \phi\left(D_{2}, \varrho i\right)>i^{-(n-2)} \phi\left(D_{1}, \varrho i\right) .
\end{aligned}
$$

Thus, similar to the proof of Lemma 4.4, we know the result holds.

Hence, for the non-bipartite unicyclic graphs, we can give

Lemma 4.6 Let $G \in \mathcal{U}_{n}(2 k+1)$ and $G^{\sigma} \in \mathfrak{D}(G), D_{3}^{n-3} \in \mathfrak{D}\left(C_{3}^{n-3}\right)$. Then

$$
\varrho\left(G^{\sigma}\right) \leq \varrho\left(D_{3}^{n-3}\right)=\sqrt{\frac{n+\sqrt{n^{2}-4 n+12}}{2}} .
$$

The equality holds if and only if $G \cong C_{3}^{n-3}$.

Proof By Lemmas 4.4 and 4.5, we know $\varrho\left(G^{\sigma}\right) \leq \varrho\left(D_{3}^{n-3}\right)$, and the equality holds if and only if $G \cong C_{3}^{n-3}$.

Also, by Theorem 3.1, we have

$$
\phi\left(D_{3}^{n-3}, \lambda\right)=\lambda^{n-4}\left(\lambda^{4}+n \lambda^{2}+n-3\right)
$$

and then

$$
\varrho^{2}\left(D_{3}^{n-3}\right)=\frac{n+\sqrt{n^{2}-4 n+12}}{2} .
$$

Thus the result holds.

Now, it is sufficient to compare the skew-spectral radius of $D_{3}^{n-3}$ and the adjacency spectral radius of $C_{4}^{n-4}$.

Lemma 4.7 If $n>5$, then $\rho\left(C_{4}^{n-4}\right)<\varrho\left(D_{3}^{n-3}\right)$.

Proof By Lemmas 4.3 and 4.6, we have

$$
\varrho^{2}\left(D_{3}^{n-3}\right)=\frac{n+\sqrt{n^{2}-4 n+12}}{2} .
$$


And

$$
\rho^{2}\left(C_{4}^{n-4}\right)=\frac{n+\sqrt{n^{2}-8 n+32}}{2} .
$$

It is easy to know that $\rho\left(C_{4}^{n-4}\right)<\varrho\left(D_{3}^{n-3}\right)$ when $n>5$.

Finally, by the above results, we can get the main result of this section.

Theorem 4.1 Let $G$ be a unicyclic graph of order $n, G^{\sigma} \in \mathfrak{D}(G)$. If $n>5$, then

$$
\varrho\left(G^{\sigma}\right) \leq \varrho\left(D_{3}^{n-3}\right)=\sqrt{\frac{n+\sqrt{n^{2}-4 n+12}}{2}} .
$$

The equality holds if and only if $G \cong C_{3}^{n-3}$.

Proof By Lemmas 4.3, 4.6 and 4.7, we know the result holds.

Remark 4.1 For the adjacency spectral radius of a unicyclic graph, it is well known that $\rho(G)<\rho\left(C_{3}^{n-3}\right)$ for $G \varsubsetneqq C_{3}^{n-3}$. It is interesting that for the skew-spectral radius of an oriented unicyclic graph, we also have $\varrho\left(G^{\sigma}\right)<\varrho\left(D_{3}^{n-3}\right)(n>5)$ for $G \supsetneqq C_{3}^{n-3}$. But for $n=4,5$, the inequality does not hold. In fact, by making a simple comparison, we know that $\varrho\left(D_{3}^{1}\right)<\rho\left(C_{4}\right)$ and $\varrho\left(D_{3}^{2}\right)=\rho\left(C_{4}^{1}\right)$.

\section{Competing interests}

The author declares that he has no competing interests.

\section{Acknowledgements}

This work was supported by the National Natural Science Foundation of China (No. 11171373).

Received: 11 February 2012 Accepted: 18 September 2012 Published: 2 October 2012

\section{References}

1. Cvetković, D, Doob, M, Sachs, H: Spectra of Graphs. Academic Press, New York (1980)

2. Godsil, CD: Algebraic Combinatorics. Chapmam \& Hall, London (1993)

3. Horn, R, Johnson, C: Matrix Analysis. Cambridge University Press, Cambridge (1989)

4. Tutte, WT: The factorization of linear graphs. J. Lond. Math. Soc. 22, 107-111 (1947)

5. Lovász, L, Plummer, M: Matching Theory. Ann. of Discrete Math., vol. 29. North-Holland, New York (1988)

6. Robertson, N, Seymour, PD, Thomas, R: Permanents, Pfaffian orientations and even directed circuits. Ann. Math. 150, 929-975 (1999)

7. Yan, WG, Zhang, FJ: Enumeration of perfect matchings of a type of Cartesian products of graphs. Discrete Appl. Math. $154,145-157(2006)$

8. IMA-ISU research group on minimum rank: Minimum rank of skew-symmetric matrices described by a graph. Linear Algebra Appl. 432, 2457-2472 (2010)

9. Shader, B, So, WS: Skew spectra of oriented graphs. Electron. J. Comb. 16, N32 (2009)

10. Adiga, C, Balakrishnan, R, So, WS: The skew energy of a graph. Linear Algebra Appl. 432, 1825-1835 (2010)

11. Gong, SC, Xu, GH: The characteristic polynomial and the matchings polynomial of a weighted oriented graph. Linear Algebra Appl. 436, 3597-3607 (2012)

12. Hou, YP, Lei, TG: Characteristic polynomials of skew-adjacency matrices of oriented graphs. Electron. J. Comb. 18, P156 (2011)

13. Hong, Y: Bounds on the spectra of unicyclic graphs. J. East China Norm. Univ. 1, 31-34 (1986) (in Chinese)

doi:10.1186/1029-242X-2012-211

Cite this article as: $\mathrm{Xu}$ : Some inequalities on the skew-spectral radii of oriented graphs. Journal of Inequalities and Applications 2012 2012:211. 